\title{
TDMA-Type Preamble for Low Complexity Multi-User Synchronization in OFDMA Uplink
}

\author{
Xiaoyu Fu, Student Member, IEEE, and Hlaing Minn, Member, IEEE \\ The University of Texas at Dallas. (\{xxf031000, hlaing.minn $\} @$ utdallas.edu)
}

\begin{abstract}
The existing synchronization and channel estimation methods utilizing the IEEE 802.16a OFDMA uplink preamble structure are associated with very high complexity. This paper proposes a TDMA-type preamble structure and the corresponding synchronization and channel estimation methods for OFDMA uplink. The proposed approach decouples multi-user synchronization and channel estimation tasks into several singleuser synchronization and channel estimation tasks by which it achieves much lower complexity and better performance than the existing approaches.
\end{abstract}

\section{INTRODUCTION}

The existing uplink frequency offset estimation methods for OFDMA systems such as IEEE 802.16a [1] can be categorized into two types depending on the system conditions considered. The first one considers single unsynchronized user scenario such as [2] where it assumes that only one new user's frequency and timing offsets need to be estimated while other users have already been perfectly synchronized to the Base Station (BS) references. Hence, this approach estimates frequency and timing offsets for only one user and would not fully represent a practical situation. The second category addresses multiple unsynchronized users scenarios such as [3] and [4]. In [4], the system requires that the Subscriber Stations (SS) transmit symbols on a group of adjacent sub-carriers only and it loses frequency diversity. To gain frequency diversity, in [3] Cao considered an interleaved sub-carrier assignment and presented a subspace method to estimate multi-user frequency offsets in OFDMA uplink. However, its computation complexity is quite high and its estimation accuracy depends on the computation complexity and the number of active users.

In this paper, we focus on how to reduce the complexity in estimating the multi-user synchronization parameters at the BS for OFDMA uplink. We propose an OFDMA system with a TDMA-type preamble structure for the uplink by which low complexity estimation of the multi-user synchronization parameters at the BS is achieved. We also present frequency offset estimator, channel estimator and symbol detector corresponding to the proposed preamble structure. Compared with the existing methods, the proposed methods have the following advantages: 1) low computation complexities, 2) fast multi-user synchronization within two OFDM symbols, 3) robustness to multi-user interference, and 4) applicability with any sub-carrier assignment scheme.

\section{System Description And Proposed Preamble STRUCTURE}

An OFDMA system consists of $N$ sub-carriers which are divided into $Q$ sub-channels. Each sub-channel consists of $P=N / Q$ sub-carriers (or $P=\left(N-N_{\text {null }}\right) / Q$ if $N_{\text {null }}$ null sub-carriers are inserted). The sub-carrier assignment for each sub-channel can be 'adjacent' where a disjoint set of $P$ adjacent sub-carriers is assigned to each sub-channel or 'interleaved' where $P$ sub-carriers of a sub-channel are disjointly interleaved with the other sub-channels' sub-carriers. The length of one OFDM symbol is $N_{t}=N+N_{g}$ samples where $N$ is the length of useful part and $N_{g}$ is the length of cyclic prefix part.

In OFDMA systems, a SS first performs a coarse timing and carrier frequency synchronization based on the downlink control/broadcast channel. Then SS enters into an initial ranging process (adjustment of coarse timing and power at the SSs based on the feedback from the BS) and it will be called a ranging subscriber station (RSS). Once initial ranging process is complete, the SS (now called data subscriber station (DSS)) transmits data on one or more assigned sub-channels depending on its data rate requirement. In this paper, we consider the following scenario: the BS has already completed initial ranging process for all DSSs. Hence, the BS knows the number of total DSSs (or users) $K$ in one uplink sub-frame. Since the coarse timing and carrier frequency adjustments at the SSs are not perfect, there exist a time offset $d_{k}$ and a frequency offset $v_{k}$ (normalized by the sub-carrier spacing) for the $k$-th user.

At the beginning of each user's data packet, a preamble (training signal) is transmitted for synchronization purpose. The preamble structure for the OFDMA uplink defined in the IEEE 802.16a is shown in Fig. 1(a). The resource for the uplink preamble is all sub-carriers (except the ranging subcarriers and the null sub-carriers) over one OFDM symbol duration. Different users' training signals are orthogonal in frequency-domain if perfectly synchronized.

Our proposed preamble structure for the OFDMA uplink is presented in Fig. 1(b) and 1(c). Different from the IEEE 802.16 a preamble structure, the proposed preamble structure is of TDMA-type where different users' training signals are orthogonal in time-domain. A guard time interval is inserted between training signals of adjacent (in time) users to avoid inter-user interference in the synchronization tasks. The total resource for the preamble is one or two OFDM symbol 
duration which depends on the number of DSSs $K$ and the preamble guard time duration. The training signals are of single-carrier-type sequences in the time-domain. When only one OFDM symbol interval is used for preamble, the preamble resource is divided into two identical blocks of one half OFDM symbol duration and the two preamble blocks are separated by one OFDM data symbol as shown in Fig. 1(b). When twoOFDM-symbol interval is used for preamble, the preamble resource is simply divided into two identical blocks of one OFDM symbol duration as shown in Fig. 1(c).

Each preamble block is divided into $K$ time-slots which are assigned to $K$ users. The length of each time-slot is $M=\left\lfloor\frac{N_{t}-2 d_{\max }-2 L+2}{2 K}\right\rfloor$ samples for the structure in Fig. 1(b) and $M=\left\lfloor\frac{2 N_{t}-d_{\max }-L+1}{2 K}\right\rfloor$ for the structure in Fig. 1(c) where $\lfloor x\rfloor$ gives the maximum integer less than or equal to $x$. A guard interval of $d_{\max }-L+1$ samples is inserted between the end of preamble time-slot and the beginning of data signal. The $M$ samples of each time-slot include a guard time-interval of $d_{\max }+L-1$ samples and training signal block of $M-d_{\max }-L+1$ samples. The parameter $d_{\max }$ is the maximum timing offset (in samples) which is determined by the performance of the ranging process (the esitmation performance of the BS's timing estimator) and $L$ is the number of sample-spaced channel taps. Each user has two time-slots for preamble transmission which are separated by a distance of $\frac{3 N_{t}}{2}$ samples or $N_{t}$ samples corresponding to the preamble duration of one or two-OFDM-symbol interval, respectively. We have assumed that the BS informs the DSSs of the preamble time-slot length ${ }^{1}$ and the corresponding timeslot locations via the downlink control/broadcast channel.

By this preamble structure, the multi-user frequency offsets estimation problem is decoupled into several single user frequency offset estimation problems which can easily be tackled with low complexity. The multi-user channel estimation problem is also converted into several single user channel estimation problems and the interference in channel estimation among different users is avoided.

\section{Signal Model}

The complex baseband received signal vector over the first two-symbol duration is given by

$$
\begin{aligned}
& \boldsymbol{r}=\sum_{k=0}^{K-1} \boldsymbol{V}\left(v_{k}, 0: 2 N_{t}-1\right) \boldsymbol{A}_{k} \boldsymbol{h}_{k}+\boldsymbol{z} \\
& \text { where } \boldsymbol{r}=\left[r(0), \ldots, r\left(2 N_{t}-1\right)\right]^{T} \\
& \boldsymbol{V}\left(v_{k}, l: m\right)=\operatorname{diag}\left[e^{\frac{j 2 \pi l v_{k}}{N}}, e^{\frac{j 2 \pi(l+1) v_{k}}{N}}, \ldots, e^{\frac{j 2 \pi m v_{k}}{N}}\right] \\
& \boldsymbol{h}_{k}=\left[\mathbf{0}_{d_{k}}^{T}, h_{k}(0), \ldots, h_{k}(L-1), \mathbf{0}_{d_{\max }-d_{k}}^{T}\right]^{T} \\
& \boldsymbol{A}_{k}=\left[\begin{array}{llll}
s_{k}(0) & 0 & \ldots & 0 \\
s_{k}(1) & s_{k}(0) & \ldots & 0 \\
\vdots & \vdots & \ddots & \vdots \\
s_{k}\left(2 N_{t}-1\right) & s_{k}\left(2 N_{t}-2\right) & \ldots & s_{k}(\alpha)
\end{array}\right] .
\end{aligned}
$$

In the above equation, $\alpha=2 N_{t}-d_{\max }-L, \boldsymbol{z}$ is a vector of uncorrelated, circularly-symmetric, complex Gaussian noise

\footnotetext{
${ }^{1}$ If a simpler version is desired, a fixed-length preamble time-slot can be adopted.
}

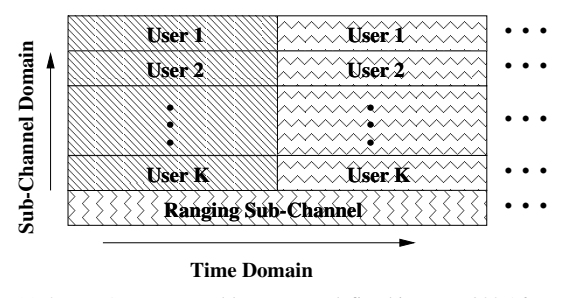

(a) OFDMA-type preamble structure defined in IEEE 802.16a

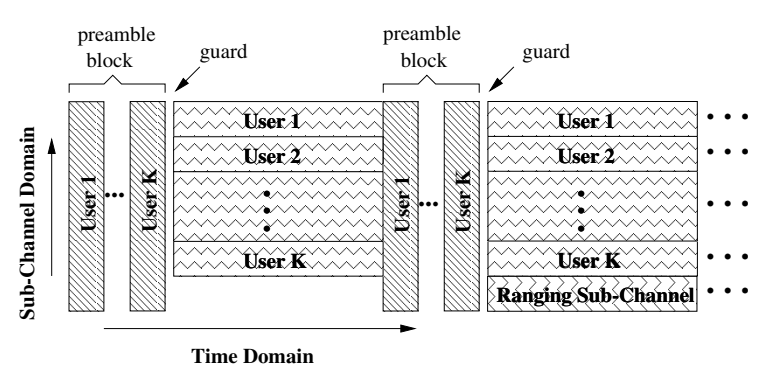

(b) TDMA-type preamble structure (one symbol duration) for OFDMA uplink

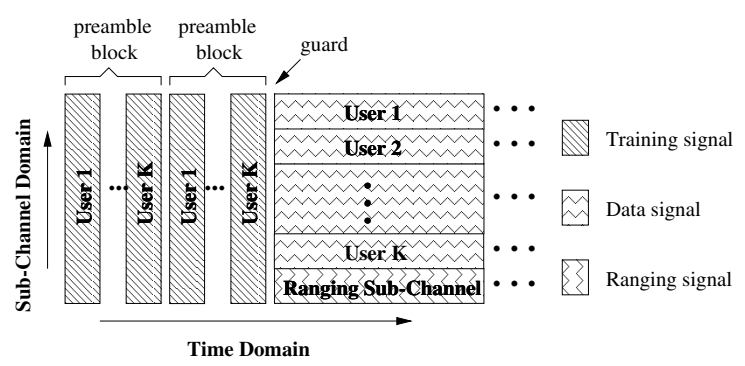

(c) TDMA-type preamble structure (two symbol duration) for OFDMA uplink

Fig. 1. Different preamble structures for OFDMA uplink

samples with zero mean and equal variance $\sigma_{w}^{2}$, and $\mathbf{0}_{p}$ is the $p \times 1$ all-zero vector. $\left\{\boldsymbol{s}_{k}(n)\right\}$ are the samples of the $k$-th DSS's transmitted signal vector which is given by

$$
\boldsymbol{s}_{k}=\left\{\begin{array}{cl}
{\left[\mathbf{0}_{p_{k}}^{T}, \boldsymbol{s}_{k, t}^{T}, \mathbf{0}_{\frac{N_{t}}{2}-k M-M}^{T}, \boldsymbol{s}_{k, d}^{T},\right.} & \\
\left.\mathbf{0}_{p_{k}}^{T}, \boldsymbol{s}_{k, t}^{T}, \mathbf{0}_{\frac{N_{t}}{2}-k M-M}^{T}\right]^{T}, & 1 \leq K \leq K_{1} \\
{\left[\mathbf{0}_{p_{k}}^{T}, \boldsymbol{s}_{k, t}^{T}, \mathbf{0}_{N_{t}-M+d_{\max }+L-1}^{T},\right.} & \\
\left.\boldsymbol{s}_{k, t}^{T}, \mathbf{0}_{N_{t}-k M-M}^{T}\right]^{T}, & K_{1}<K \leq 2 K_{1}
\end{array}\right.
$$

where $p_{k}=k M+d_{\max }+L-1$ and $K_{1}$ is the maximum number of users that can be supported if the preamble duration is one OFDM symbol interval. Since the system can support a maximum of $Q$ DSSs, we have $K \leq Q$. In general, the preamble duration needs not be integer multiple of OFDM symbol interval but we should have $M \geq 2 L+3 d_{\max }-1$ (for identifiability in estimation of channel vector $\boldsymbol{h}_{k}$ ). In the above equation, $\boldsymbol{s}_{k, t}=\left[s_{k, t}(0), s_{k, t}(1), \ldots, s_{k, t}\left(M-d_{\max }-L\right)\right]^{T}$ is a $\left(M-d_{\max }-L+1\right) \times 1$ training signal vector and $\boldsymbol{s}_{k, d}$ is the vector representing $k$-th user's OFDM data symbol inserted between the two preamble blocks and is given by

$$
\boldsymbol{s}_{k, d}=\boldsymbol{P} \boldsymbol{W}^{-1} \boldsymbol{X}_{k}
$$

where $\boldsymbol{W}$ is the $N$-point FFT matrix with $[\boldsymbol{W}]_{l, m}=$ $e^{-j 2 \pi l m / N}$ (hence, $\boldsymbol{W}^{-1}$ is the $N$-point IFFT matrix), $\boldsymbol{X}_{k}$ is $k$-th user's transmitted data vector in the frequency-domain, and $\boldsymbol{P}$ is the cyclic prefix insertion matrix. 
We assume that the channel coefficients are unchanged during one uplink burst. Note that $0 \leq d_{k} \leq d_{\max }$ for all $k$ since the BS has already completed the ranging process. For convenience in comparison of different preamble structures, we define SNR for the $i$-th user as the ratio of total transmitted training signal energy of the $i$-th user to the total noise energy within one OFDM symbol interval ( $N$ samples) as

$$
\mathrm{SNR}_{i}=\frac{2 \boldsymbol{s}_{k, t}^{H} \boldsymbol{s}_{k, t}}{N \sigma_{w}^{2}} .
$$

\section{FREQUENCY OfFSET Estimation}

We form received training signal vectors corresponding to $k$-th user's training time-slot 1 and 2 , respectively, as

$$
\begin{aligned}
& \boldsymbol{r}_{k, t}^{(1)}=\left[r\left(p_{k}+\tau\right), \ldots, r\left(p_{k}+\tau+J-1\right)\right]^{T} \\
& \boldsymbol{r}_{k, t}^{(2)}=\left[r\left(p_{k}+\tau+D\right), \ldots, r\left(p_{k}+\tau+J-1+D\right)\right]^{T}
\end{aligned}
$$

where $p_{k}=k M+L+d_{\max }-1, D=\frac{3 N_{t}}{2}$ when the preamble duration is one OFDM symbol interval, and $D=N_{t}$ when the preamble duration is two OFDM-symbol interval. Due to the timing offset $d_{k}$, the received preamble samples $\left(r\left(p_{k}\right), \ldots, r\left(p_{k}+d_{k}\right)\right)$ and $\left(r\left(p_{k}+D\right), \ldots, r\left(p_{k}+D+d_{k}\right)\right)$ are noise-only samples and hence, including them in the frequency offset estimation will degrade the estimation performance. For the frequency offset estimator to be robust against the timing offset $d_{k}\left(\leq d_{\max }\right)$, we have excluded $d_{\text {max }}$ possibly noise-only samples from each received preamble block to be used in the frequency offset estimation. In other words, $\left(r\left(p_{k}\right), \ldots, r\left(p_{k}+d_{\max }-1\right)\right)$ and $\left(r\left(p_{k}+D\right), \ldots, r\left(p_{k}+\right.\right.$ $\left.\left.D+d_{\max }-1\right)\right)$ samples are excluded and the received vectors used in frequency offset estimation are given by (9) and (10) with $\tau=d_{\max }$ and $J=M-2 d_{\max }$.

The proposed frequency offset estimation method exploits the correlation of two identical preamble parts as

$$
\hat{v}_{k}=\frac{N}{2 \pi D} \text { angle }\left(\left(\boldsymbol{r}_{k, t}^{(1)}\right)^{H} \boldsymbol{r}_{k, t}^{(2)}\right) .
$$

The frequency offset estimation range is $\left|\hat{v}_{k}\right|<\frac{N}{2 D}$. Since each DSS has adjusted its local oscillator based on the downlink control channel, the residual frequency offset would be quite small and the above range is sufficient. Note that if the distance (first sample to first sample) between the two preamble blocks is larger, the achievable frequency offset estimation MSE performance is better, provided that the frequency offset is within the range of the estimator and the channel is time-invariant over the two preamble blocks [6].

\section{Channel Estimation}

For channel estimation, we use received training signal vectors from (9) and (10) with $\tau=0$ and $J=M$. They can be expressed as

$$
\begin{aligned}
& \boldsymbol{r}_{k, t}^{(1)}=\boldsymbol{V}\left(v_{k}, l_{1}: m_{1}\right) \boldsymbol{A}_{k, t} \boldsymbol{h}_{k}+\boldsymbol{z}_{k, t}^{(1)} \\
& \boldsymbol{r}_{k, t}^{(2)}=\boldsymbol{V}\left(v_{k}, l_{2}: m_{2}\right) \boldsymbol{A}_{k, t} \boldsymbol{h}_{k}+\boldsymbol{z}_{k, t}^{(2)}
\end{aligned}
$$

where $l_{1}=p_{k}, m_{1}=p_{k}+J-1, l_{2}=p_{k}+D, m_{2}=$ $p_{k}+J-1+D$, and

$$
\boldsymbol{A}_{k, t}=\left[\begin{array}{llll}
s_{k, t}(0) & 0 & \ldots & 0 \\
s_{k, t}(1) & s_{k, t}(0) & \ldots & 0 \\
\vdots & \vdots & \ddots & \vdots \\
s_{k, t}(J-1) & s_{k, t}(J-2) & \ldots & s_{k, t}(\lambda)
\end{array}\right] .
$$

In the above equations, $\lambda=J-d_{\max }-L, \boldsymbol{A}_{k, t}$ is the training matrix for the $k$-th user (the same for both time-slots) and $\boldsymbol{z}_{k, t}^{m}$ is a Gaussian noise vector within $k$-th user's $m$-th preamble time-slot.

First, we perform frequency offset compensation and then average the two resulting training vectors from the two timeslots (with the aim of complexity reduction) as

$$
\overline{\boldsymbol{r}}_{k, t}=\frac{1}{2}\left(\boldsymbol{V}\left(-\hat{v}_{k}, l_{1}: m_{1}\right) \boldsymbol{r}_{k, t}^{(1)}+\boldsymbol{V}\left(-\hat{v}_{k}, l_{2}: m_{2}\right) \boldsymbol{r}_{k, t}^{(2)}\right)
$$

where $\boldsymbol{V}\left(-\hat{v}_{k}, l: m\right)$ is the frequency offset compensation matrix formed by using the frequency offset estimate $\hat{v}_{k}$. Then the channel impulse response estimate $\hat{\boldsymbol{h}}_{k}$ is obtained by

$$
\hat{\boldsymbol{h}}_{k}=\left(\boldsymbol{A}_{k, t}^{H} \boldsymbol{A}_{k, t}\right)^{-1} \boldsymbol{A}_{k, t}^{H} \overline{\boldsymbol{r}}_{k, t} .
$$

Note that our signal model uses extended matrices and vectors (e.g., $\boldsymbol{h}_{k}$ is prefixed and suffixed with zeros). This extended signal model accommodates different timing offsets. Consequently, the corresponding channel estimation is robust to timing offsets. For example, we can express $\hat{\boldsymbol{h}}_{k}$ as

$$
\hat{\boldsymbol{h}}_{\boldsymbol{k}}=\left[n_{1}, \ldots, n_{d_{k}}, \hat{h}_{k}(0), \ldots, \hat{h}_{k}(L-1), n_{d_{k+1}}, \ldots, n_{d_{\max }}\right]^{T}
$$

where $n_{1}, n_{2}, \ldots, n_{d_{\max }}$ are noise terms. Since $0 \leq d_{k} \leq$ $d_{\text {max }}$, we can see from (17) that the channel estimation vector captures the actual channel taps in the presence of all possible timing offsets (after ranging process).

\section{Symbol Detection}

In the following, we discuss data detection for the first OFDM data symbol. Detection for the other data symbols follows the same. The received data signal vector after the cyclic prefix removal is given by

$$
\boldsymbol{r}_{d}=[r(a), \ldots, r(b)]^{T}
$$

where $a=N_{g}+\frac{N_{t}}{2}, b=N_{g}+\frac{N_{t}}{2}+N-1$ for the preamble duration of one OFDM symbol interval and $a=N_{g}+2 N_{t}$, $b=N_{g}+2 N_{t}+N-1$ for the preamble duration of two OFDM symbol interval.

In the presence of the frequency and timing offsets ${ }^{2}$, the received data vector in the time-domain can be given by

$$
\begin{gathered}
\boldsymbol{r}_{d}=\sum_{k=0}^{K-1} \boldsymbol{V}\left(v_{k}, a: b\right) \boldsymbol{W}^{-1} \tilde{\boldsymbol{H}}_{k} \boldsymbol{X}_{k}+\boldsymbol{z}_{d} \\
=\sum_{k=0}^{K-1} \boldsymbol{V}\left(v_{k}, a: b\right) \boldsymbol{W}^{-1} \tilde{\boldsymbol{H}}_{k} \boldsymbol{\varphi}_{k} \boldsymbol{X}+\boldsymbol{z}_{d}=\boldsymbol{B} \boldsymbol{X}+\boldsymbol{z}_{d}(19) \\
\text { where } \boldsymbol{X}=\sum_{k=0}^{K-1} \boldsymbol{X}_{k} \\
\boldsymbol{B}=\sum_{k=0}^{K-1} \boldsymbol{V}\left(v_{k}, a: b\right) \boldsymbol{W}^{-1} \tilde{\boldsymbol{H}}_{k} \boldsymbol{\varphi}_{k} \\
\tilde{\boldsymbol{H}}_{k}=\operatorname{diag}\left[\boldsymbol{W} \boldsymbol{h}_{k}\right] \equiv \operatorname{diag}\left[\boldsymbol{H}_{k}\right]
\end{gathered}
$$

\footnotetext{
${ }^{2}$ The guard interval is designed to satisfy $d_{\max } \leq N_{g}-L-1$.
} 
$\boldsymbol{z}_{d}$ is the Gaussian noise vector and $\boldsymbol{\varphi}_{k}$ is the sub-carrier assignment matrix for the $k$-th user given by

$$
\begin{aligned}
\varphi_{k} & =\operatorname{diag}\left[\varphi_{k}(0), \ldots, \varphi_{k}(N-1)\right] \\
\varphi_{k}(m) & =\left\{\begin{array}{cc}
1 & \text { if } m \text {-th sub-carrier is assigned to } k \text {-th user } \\
0 & \text { otherwise. }
\end{array}\right.
\end{aligned}
$$

The transmitted data vector $\boldsymbol{X}$ (for all DSSs) in the frequencydomain can be detected by using the least-squares method as

$$
\hat{\boldsymbol{X}}=\left(\hat{\boldsymbol{B}}^{H} \hat{\boldsymbol{B}}\right)^{-1} \hat{\boldsymbol{B}}^{H} \boldsymbol{r}_{d}
$$

where $\hat{\boldsymbol{B}}$ is the matrix formed by using frequency offset estimate $\hat{v}_{k}$ and channel impulse response estimate $\hat{\boldsymbol{h}}_{k}$ in (21). Note that the information of exact timing point is not required in our symbol detection. In other words, it is robust to timing estimation errors as explained below. In general, timing offsets can considerably degrade the channel estimation [8]. However, we have used the extended channel impulse response vectors which incorporate possible timing offsets, and hence, possible degradation in channel estimation due to timing offsets is avoided. Consequently, symbol detection which uses channel estimates is not affected by timing offsets.

\section{Simulation Results and Discussions}

\section{A. Simulation Parameters}

In this section, we compare the proposed training structure with the IEEE 802.16a training structure defined in [1] in terms of performance in frequency offset estimation, channel impulse response estimation and symbol detection.

The OFDMA system parameters are selected from [1]. The total number of sub-carriers $N$ is 2048 which are divided into $Q=32$ sub-channels. The maximum time offset $d_{\max }$ is 32 samples. Guard interval $N_{g}$ is 64 . The uplink bandwidth is 3 $\mathrm{MHz}$ and sub-carrier spacing is $1.67 \mathrm{KHz}$. To be consistent with [3], no null sub-carrier is inserted in the simulation. The multiplexing mode is TDD. For simplicity, we use BPSK format data transmission. The combined transmit and receive filter is a raised-cosine filter $g_{T}(t)$ with a roll-off factor of 0.5 . The SUI-3 channel model with 3 paths [7] is considered and the number of sample-spaced channel taps is $L=7$.

\section{B. Algorithms for the IEEE 802.16a Training Structure}

We consider the first two OFDM symbols in one uplink burst with the OFDM-type training structure defined in [1]. The first is training symbol and the second is data symbol. Let $r$ denote the vector which contains the first two received symbols after the cyclic prefix removal. Then

$$
\begin{aligned}
\boldsymbol{r} & =\left[\boldsymbol{r}_{t}^{T}, \boldsymbol{r}_{d}^{T}\right]^{T} \\
\boldsymbol{r}_{t} & =\sum_{k=0}^{K-1} \boldsymbol{V}\left(v_{k}, N_{g}: N_{t}-1\right) \boldsymbol{W}^{-1} \tilde{\boldsymbol{X}}_{k, t} \boldsymbol{H}_{k}+\boldsymbol{z}_{t} \\
= & \sum_{k=0}^{K-1} \boldsymbol{V}\left(v_{k}, N_{g}: N_{t}-1\right) \boldsymbol{W}^{-1} \tilde{\boldsymbol{X}}_{k, t} \boldsymbol{\varphi}_{k} \boldsymbol{H}+\boldsymbol{z}_{t} \\
\text { where } \quad \boldsymbol{H} & =\sum_{k=0}^{K-1} \boldsymbol{H}_{k}^{\prime} \\
\boldsymbol{H}_{k}^{\prime} & =\boldsymbol{\varphi}_{k} \boldsymbol{H}_{k},
\end{aligned}
$$

and $\boldsymbol{r}_{t}, \boldsymbol{r}_{d}$, and $\boldsymbol{z}_{t}$ are $N \times 1$ vectors of training, data, and Gaussian noise, respectively. We choose the frequency estimator from [3] since it can be used in multi-user scenario with the interleaved sub-carrier assignment. For channel impulse response estimation, the LS estimator is applied as

$$
\begin{aligned}
\hat{\boldsymbol{H}} & =\left(\boldsymbol{C}^{H} \boldsymbol{C}\right)^{-1} \boldsymbol{C}^{H} \boldsymbol{r}_{t} \\
\text { where } \quad \boldsymbol{C} & =\sum_{k=0}^{K-1} \boldsymbol{V}\left(\hat{v}(k), N_{g}: N_{t}-1\right) \boldsymbol{W}^{-1} \tilde{\boldsymbol{X}}_{k, t} \boldsymbol{\varphi}_{k}(32)
\end{aligned}
$$

and $\tilde{\boldsymbol{X}}_{k, t}$ is the known diagonal matrix representing the training symbol in the frequency-domain for the $k$-th user. Note that $\hat{\boldsymbol{H}}$ consists of $K$ users' channel frequency responses. The proposed method in (16) uses the knowledge of channel length $L$ and the maximum timing offset $d_{\max }$. To be a fair comparison, we incorporate the knowledge of $L$ and $d_{\max }$ into the LS channel estimator of (31) as follows: We separate the $N \times 1$ vector $\hat{\boldsymbol{H}}$ into $K$ vectors $\left\{\hat{\boldsymbol{H}}^{\prime}{ }_{k}\right\}$, where each vector contains $P$ non-zero elements whose locations are defined by $\varphi_{k}$ (c.f. (30)). The channel impulse response estimate is then obtained as $\hat{\boldsymbol{h}}_{k}=\boldsymbol{\Omega}_{k} \hat{\boldsymbol{H}}^{\prime}{ }_{k}$ where $\boldsymbol{\Omega}_{k}=\left(\boldsymbol{F}_{0}^{H} \boldsymbol{\varphi}_{k} \boldsymbol{F}_{0}\right)^{-1} \boldsymbol{F}_{0}^{H}$ and $\boldsymbol{F}_{0}$ is the first $d_{\max }+L$ columns of the $N$-point FFT matrix $\boldsymbol{F}$. The final estimate of $\boldsymbol{H}^{\prime}{ }_{k}$ is obtained as $\boldsymbol{\varphi}_{k} \boldsymbol{F} \hat{\boldsymbol{h}}_{k}$. For symbol detection, we use the same LS method as (25) where $a=N_{g}+N_{t}$ and $b=N_{g}+N_{t}+N-1$ due to the different preamble structure.

\section{Computation Complexity}

The proposed frequency offset estimator uses only correlation of training signal and it requires $K\left(M-d_{\max }-L+1\right)$ complex products, $K\left(M-d_{\max }-L\right)$ complex additions, $K$ real products and $K$ angle operations. So its complexity is much smaller than the reference method [3] which applied MUSIC method and required $K U\left(Q^{2}+Q+1\right)+Q N$ complex products, $K U\left(Q^{2}-1\right)+Q N-Q$ complex additions, one real product, a SVD computation for a $Q \times Q$ matrix, and finding the minimum among $U$ values for each user. $Q$ is the maximum number of users the system can support and $U$ is the number of frequency offset candidate points within the estimation range for each user.

In the proposed channel estimation method, only the terms $\boldsymbol{V}\left(\hat{v}_{k}, l_{i}: m_{i}\right) \boldsymbol{r}_{k, t}^{(i)}$ need to be refreshed each time since the term $\left(\boldsymbol{A}_{k, t}^{H} \boldsymbol{A}_{k, t}\right)^{-1} \boldsymbol{A}_{k, t}^{H}$ is fixed for each user (see (16)). On the other hand, for the LS method defined in (31) for the IEEE 802.16a preamble, the term $\left(\boldsymbol{C}^{H} \boldsymbol{C}\right)^{-1} \boldsymbol{C}^{H}$ needs to be computed each time. Hence, the channel estimation complexity in the proposed approach is much smaller than that in the reference approach. For the symbol detector, the complexities of the proposed method and the reference method are the same since both use the same LS method.

\section{Simulation Results}

First, we compare the performance of the proposed TDMAtype preamble based frequency offset estimator with that of the MUSIC method proposed in [3]. We evaluate these two algorithms for $K=5,15$, and 25 . The simulation results for the square-root of mean-square error (RMSE) of the 


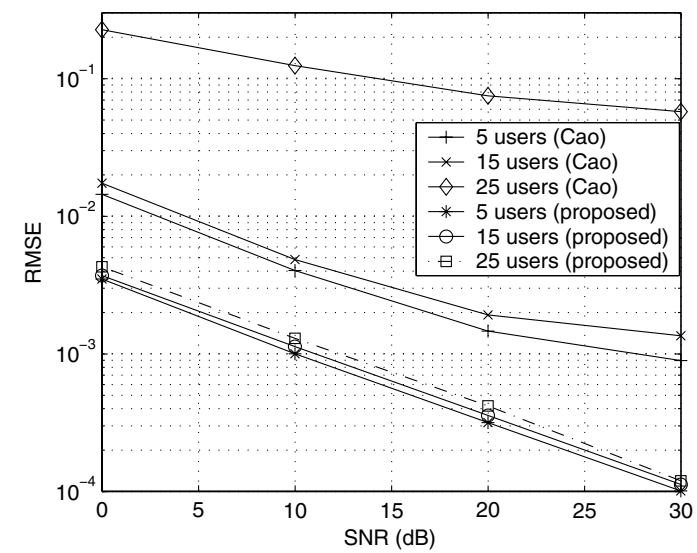

Fig. 2. The RMSE of frequency offset estimators

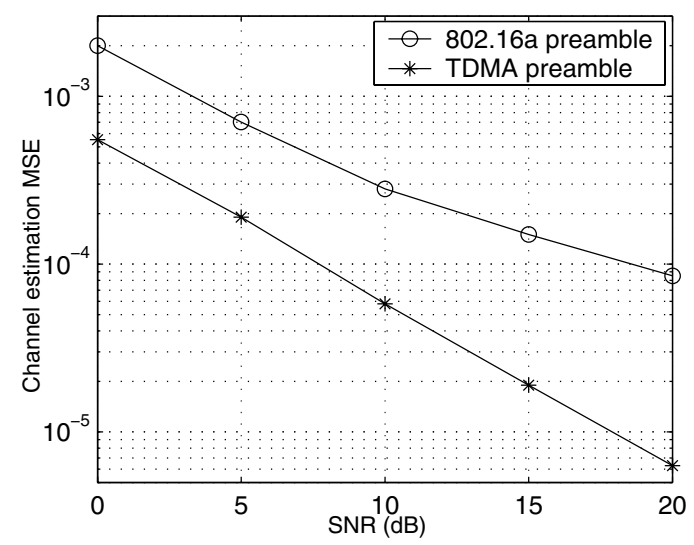

Fig. 3. The MSE of channel impulse response estimaton

normalized frequency offset estimation are shown in Fig. 2. We observe that the estimation performance of the MUSIC method from [3] depends on the number of users $K$ and degrades largely when $K$ is close to $Q$, the maximum number of users the system can support. The performance of the TDMA-type structure based estimator is not only better than the method from [3] but also not degraded when $K$ increases.

Fig. 3 shows the MSE of the channel impulse response estimators obtained with the TDMA-type preamble and the IEEE 802.16a uplink preamble. The number of users $K$ is 8. To decouple the effects of frequency offset errors, perfect frequency recovery is assumed in evaluating both channel estimation methods. From Fig. 3, it can be observed that the channel estimation performance of the proposed approach achieves about $5 \mathrm{~dB}$ improvement.

Finally, we compare the performance of the symbol detector obtained with the proposed TDMA-type preamble and the 802.16a preamble in the presence of frequency and timing offsets. From Fig. 4, we can see that the uncoded BER of the proposed preamble based detector is about $1 \mathrm{~dB}$ better than the 802.16a preamble based method, and it is quite close to the ideal case with perfect synchronization and channel estimation.

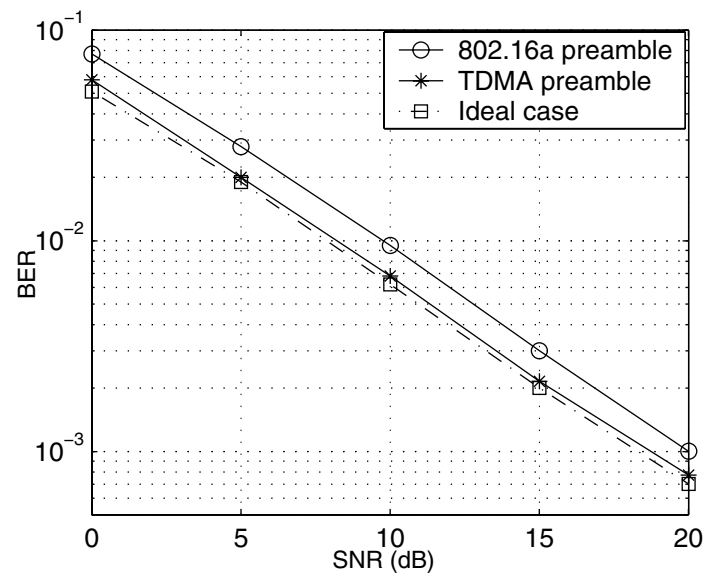

Fig. 4. The uncoded BER comparison

\section{CONCLUSIONS}

Synchronization, channel estimation, and detection in OFDMA uplink typically require a very large complexity and accomplishing these tasks with low complexity is a challenging issue. In this paper, we addressed this issue and proposed a TDMA-type training structure and the corresponding synchronization and channel estimation methods for OFDMA uplink. The proposed approach decouples multiuser synchronization and channel estimation tasks into several single-user synchronization and channel estimation tasks by which it achieves much lower complexity, better performance, and faster synchronization/channel estimation than the existing approaches. The proposed preamble structure also achieves a much greater robustness to multi-user interference.

\section{ACKNOWLEDGMENT}

This work was supported in part by the Erik Jonsson School Research Excellence Initiative, the University of Texas at Dallas, Texas, USA.

\section{REFERENCES}

[1] IEEE LAN/MAN Standards Committee, "Broadband Wireless Access: IEEE MAN standard," IEEE 802.16 a, 2003.

[2] M. Morelli, "Timing and Frequency Synchronization for the Uplink of an OFDMA System,' IEEE Trans. Commun., Vol. 52, No. 2, Feb, 2004, pp. 296-306.

[3] Z. Cao, U. Tureli, Y.D. Yao, "Efficient Structure-Based Carrier Frequency Offset Estimation for Interleaved OFDMA Uplink," Proc. IEEE ICC, Vol. 5, pp.3361 - 3365, 11-15 May 2003.

[4] J.J.van de Beek, P. O. Borjesson, M. L. Bouchest, D. Landstram, J.M. Arenas, P. Odling, C. Ostberg, M. Wahlqvist, and S.K.Wilson, "A Time and Frequency Synchronization Scheme for Multiuser OFDM,' IEEE J. Select. Areas Commun., Vol 17, pp. 1900-1914, Nov.1999.

[5] M. Speth, S. A.Fechtel, G. Fock, and H. Meyr, "Optimum Receiver Design for Wireless Broad-Band Systems Using OFDM-Part 1”, IEEE Trans. Commun., Vol. 47, No.11, Nov, 1999, pp.1668-1677.

[6] H. Minn, S. Xing, and V. K. Bhargava, "Optimal Periodic Training Signal for Frequency Offset Estimation in Frequency-Selective Fading Channels," IEEE ICC, pp. 488-492, June 20-24, 2004.

[7] IEEE LAN/MAN Standards Committee, "Channel Models for Fixed Wireless Applications," Document IEEE.802.16.3c-01/29r4.

[8] H. Minn, V. K. Bhargava and K. B. Letaief, "A Combined Timing and Frequency Synchronization and Channel Estimation for OFDM," IEEE ICC, pp. 872-876, June 20-24, 2004. 